\title{
A case of extensive synovial involvement by tophaceous gout
}

Nausheen Khan, MB BS, FCRad (D)
Irma van de Werke, MB ChB, FRCR
Farzanah Ismail, MB ChB, FCRad (D)
Department of Radiology, Kalafong Hospital, Pretoria, and University of
Pretoria
Peter F Levay, MSC, MMed (Int)
Department of Internal Medicine, Kalafong Hospital

\section{Abstract}

Gout is the most common form of microcrystal arthropathy that results in deposition of uric acid crystals in and around the joints and soft tissues. The most common cause is decreased uric acid clearance by the kidneys. The radiological manifestations of gout are generally well known and have remained unchanged. We describe a case of chronic tophaceous gout with diffuse extensive involvement of the synovium around the knees bilaterally.

\section{Case report}

A 50-year-old hypertensive Indian man, known to have had chronic gout for the past 19 years, presented with clinically large, hard, nodular masses around the knees with limitation in the range of movement. He had features of metabolic syndrome (hypertensive, impaired fasting glucose, increased abdominal girth, increased triglyceride level), with hyperuricaemia. His serum uric acid levels were $7.2 \mathrm{mg} / \mathrm{dl}$ with suboptimal therapeutic response to allopurinol and anti-inflammatory drugs. Other laboratory investigations showed an erythrocyte sedimentation rate (ESR) of $10 \mathrm{~mm} / \mathrm{hr}$, rheumatoid factor $<11 \mathrm{IU} / \mathrm{l}$, and normal antinuclear antibody titre. A 24-hour urine test showed uric acid to be $2.4 \mathrm{mg} / \mathrm{dl}$. The B2 glycoproteins, anticardiolipin and antidouble stranded DNA antibody titres were within normal range.

The following radiological examinations were performed:

- anteroposterior radiograph of the feet (Fig. 1) demonstrates characteristic first metatarsophalangeal (MTP) joint involvement on the right with eccentric, dense soft-tissue swelling and para-articular punched-out erosions with overhanging margins. The sub-articular lucencies most probably represent intra-osseous tophi. There is also

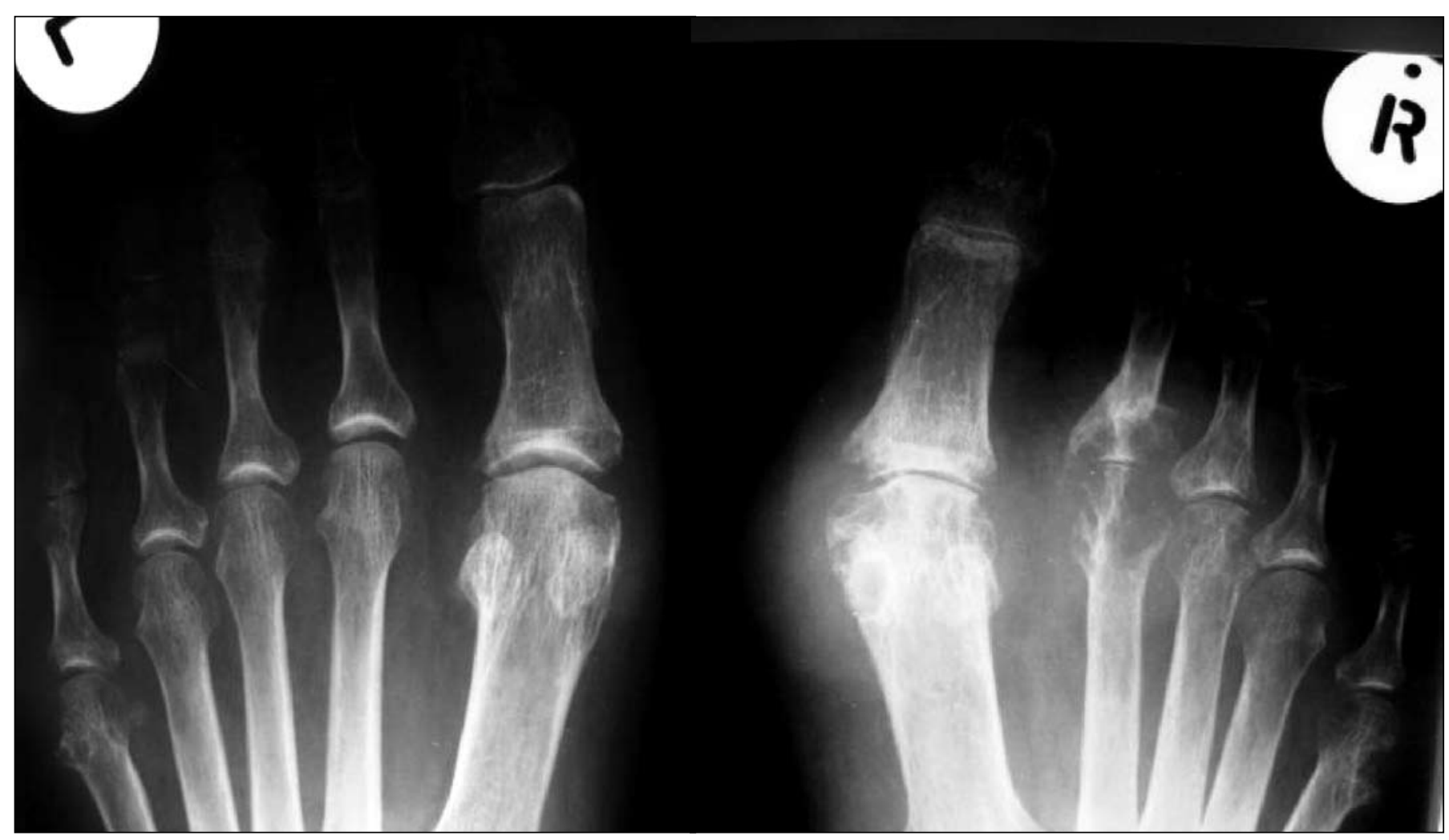

Fig. 1. Anteroposterior radiograph of the feet demonstrates first MTP joint involvement on the right. Note the eccentric, dense soft-tissue swelling and para-articular erosions. The sub-articular lucencies represent intra-osseous tophi. There is also involvement of the second, third and fifth metatarsals on the right, and fifth MTP joint on the left. 


\section{CASE REPORT}

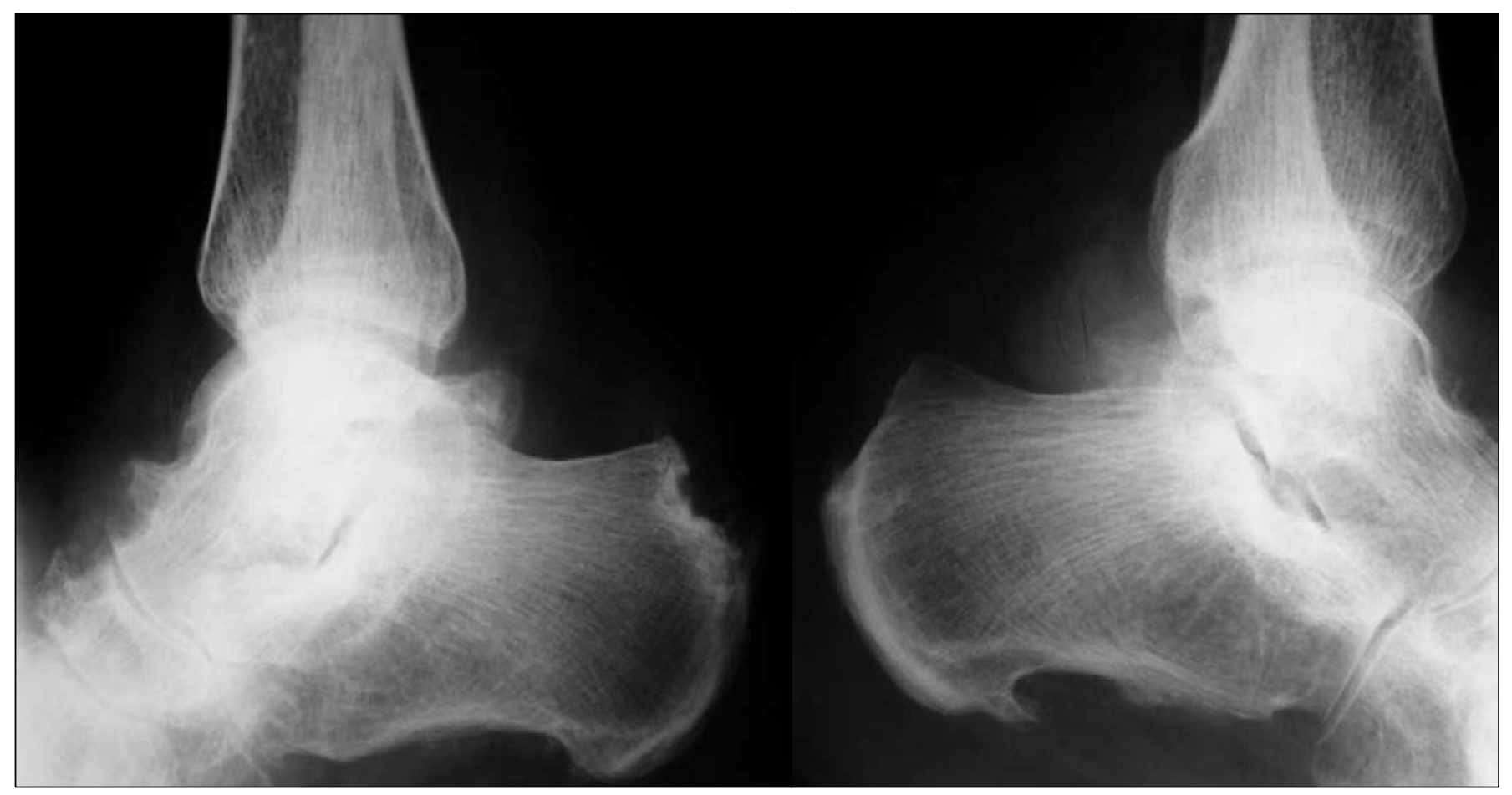

Fig. 2. Lateral radiograph of both ankles shows nodular density in the posterior recess of the left ankle joint in keeping with tophaceous deposits, and pressure erosion of the right talo-navicular joint and postero-superior aspect of the right calcaneus by soft-tissue tophi.

extensive involvement of the second, third and fifth metatarsals on the right and fifth MTP joints on the left. There is lack of osteopenia. - lateral radiograph of both ankles (Fig. 2) shows increased nodular density in the posterior recess of the ankle joint in keeping with tophaceous deposits on the left. There is also pressure erosion of the right talo-navicular joint and posterosuperior aspect of the right calcaneus by overlying soft-tissue tophi. - anteroposterior radiograph of the hands (Fig. 3) shows diffuse asymmetrical involvement of metacarpophalyngeal and interphalangeal joints. The wrists are not involved.

- anteroposterior and lateral radiographs of both knees (Fig. 4) show increased dense soft-tissue swelling around the joint, with extension into the supra and infra patella bursae and posterior aspect of the joint. There are characteristic gouty erosions and chondrocalcinosis with secondary osteoarthritis.

The differential diagnosis at this point was possible tophaceous involvement of the synovium in a patient with known gout. A mass of synovial origin was also considered; however, bilateral disease was unlikely. A non-contrast computed tomography (CT) scan was performed (Fig. 5) of both knees that showed diffuse lobulated involvement of the entire synovium by tophaceous deposits that had a Hounsfield value of $160-175$. This extensive involvement has not been previously described.

\section{Discussion}

The word gout comes from the Latin gutta (drop), in keeping with the early belief that an acute attack of the disease was a result of a poisonous body fluid dropping into the joints.' Gout has traditionally been classified as primary and/or secondary; however, this distinction has become blurred with improved understanding of the pathological bases of the various forms of the disease. Clinical progression of the disease is best described in 4 clinical phases: ${ }^{22}$

- asymptomatic hyperuricaemia

- acute gouty arthritis

- intercritical gout

- chronic tophaceous gout.

The peak incidence is between 30 and 50 years, with a male:female ratio of 5:1. The prevalence of the disease increases with age. ${ }^{24}$ Tophaceous gout, however, occurs in less than $10 \%$ of cases.

\section{Asymptomatic hyperuricaemia}

Increased levels of uric acid are found years before the onset of symptoms, and there is no evidence to suggest that treatment is warranted for asymptomatic hyperuricaemia. ${ }^{23}$

\section{Acute gouty arthritis}

This is the most common manifestation of gout, as acute inflammation owing to precipitation of urate crystals within the joint. The arthritis is initially monoarticular; as the disease progresses, more joints may be involved. The first joint to be involved is usually (50\% of cases) the MTP joint.

\section{Intercritical gout}

The disease is asymptomatic; the patient has persistent hyperuricaemia, and synovial fluid analysis shows monosodium urate (MSU) crystals. 


\section{CASE REPORT}
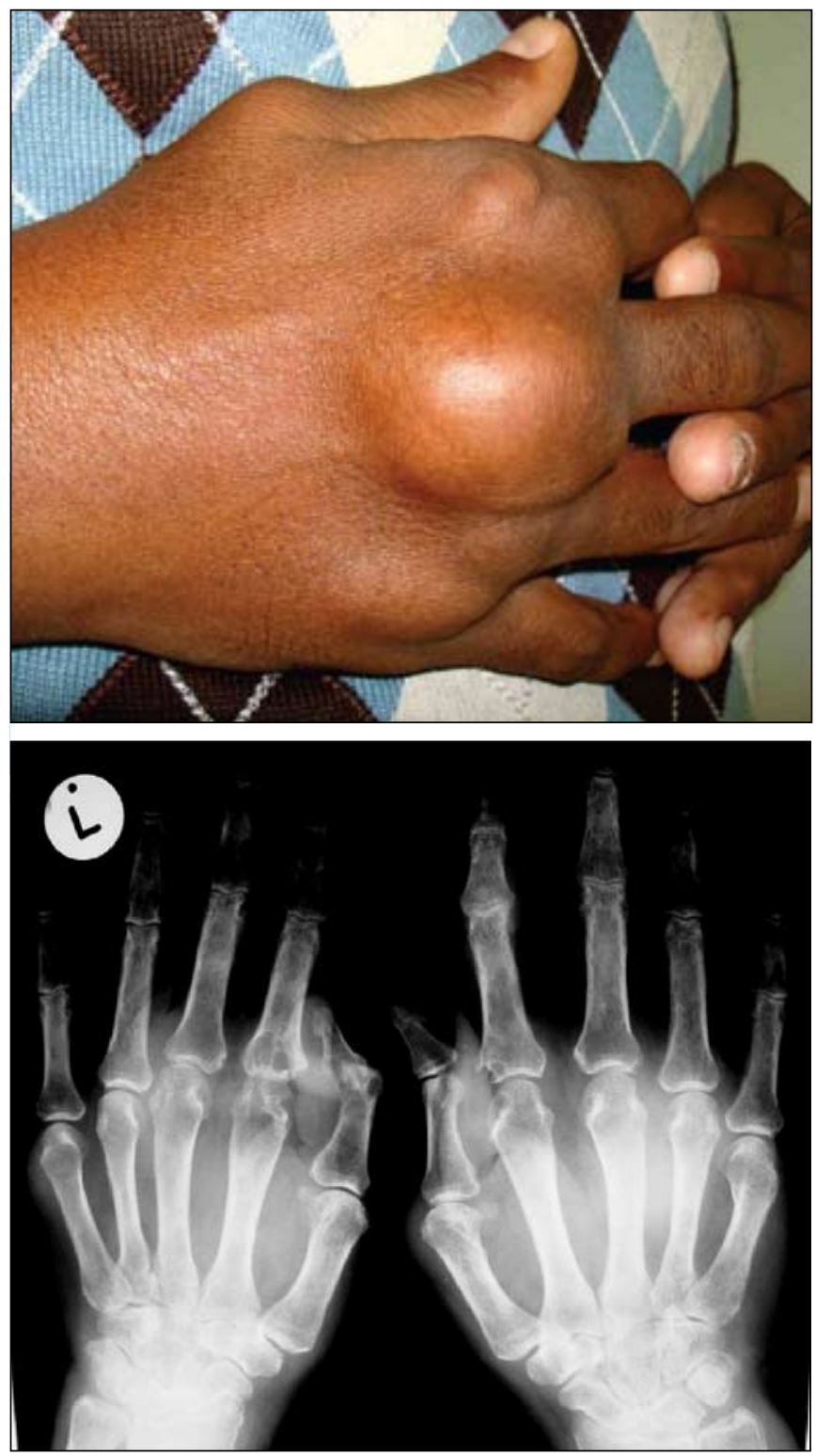

Fig. 3. Photograph of the right hand and anteroposterior radiograph of both hands show large soft-tissue tophi around the MTP joints and typical involvement of the MTP joints by gout.

\section{Chronic tophaceous gout}

In this form, the disease is established with deposition of MSU crystals in the tendons, ligaments, cartilage, bone and soft tissues including bursae and other synovial spaces. ${ }^{13}$ Although tophi can be deposited within a joint, the imaging characteristics of such intra-articular tophi are rarely reported and not well known. ${ }^{5}$ Tophaceous deposits may mimic space-occupying lesions and occasionally be confused with rheumatoid nodules. ${ }^{6} \mathrm{MSU}$ crystals do not calcify. These opaque crystal deposits are composed of calcium pyrophosphate dehydrate, reflecting an association with pseudo-gout.
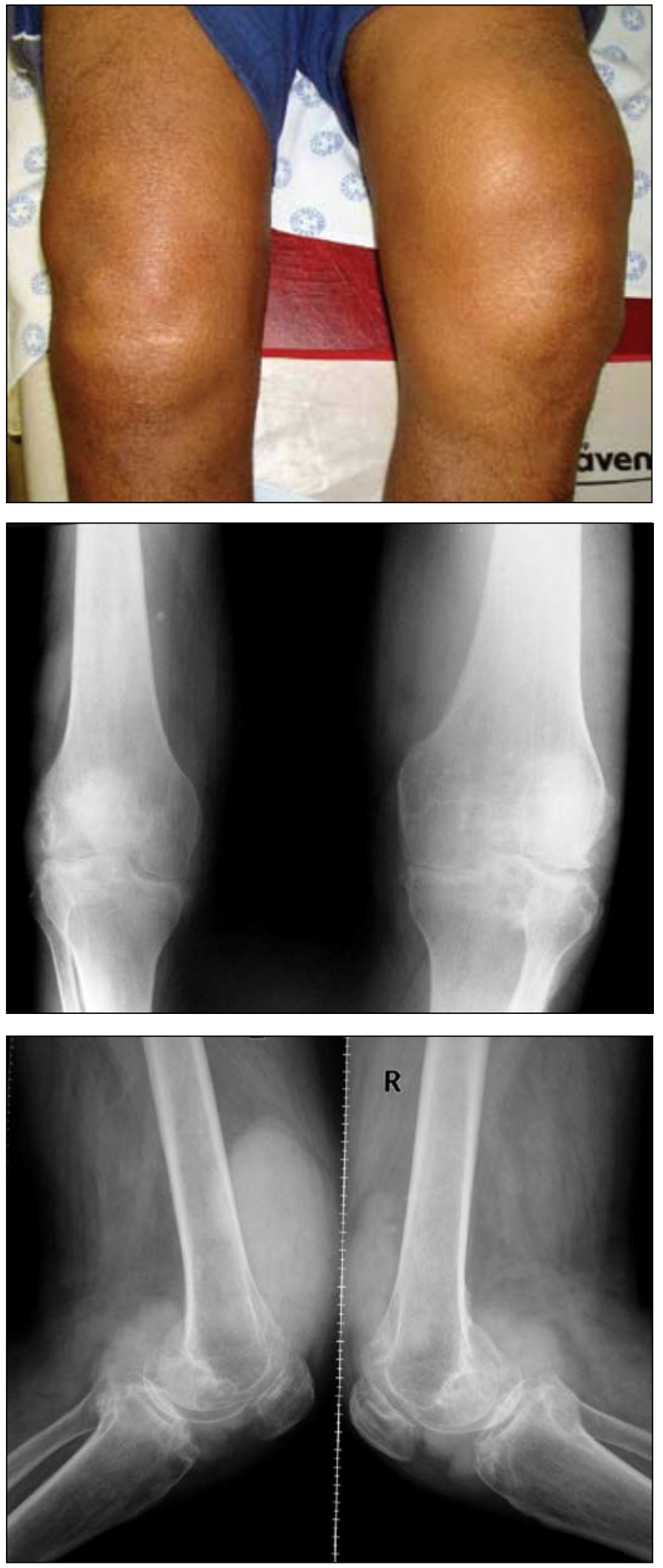

Fig. 4. Photograph of both knees shows extensive tophi. Accompanying anteroposterior and lateral radiographs show dense soft-tissue swelling around the joint, with extension into the supra and infra patella bursae and posterior aspect of the joint. Also note typical gouty erosions. 


\section{CASE REPORT}

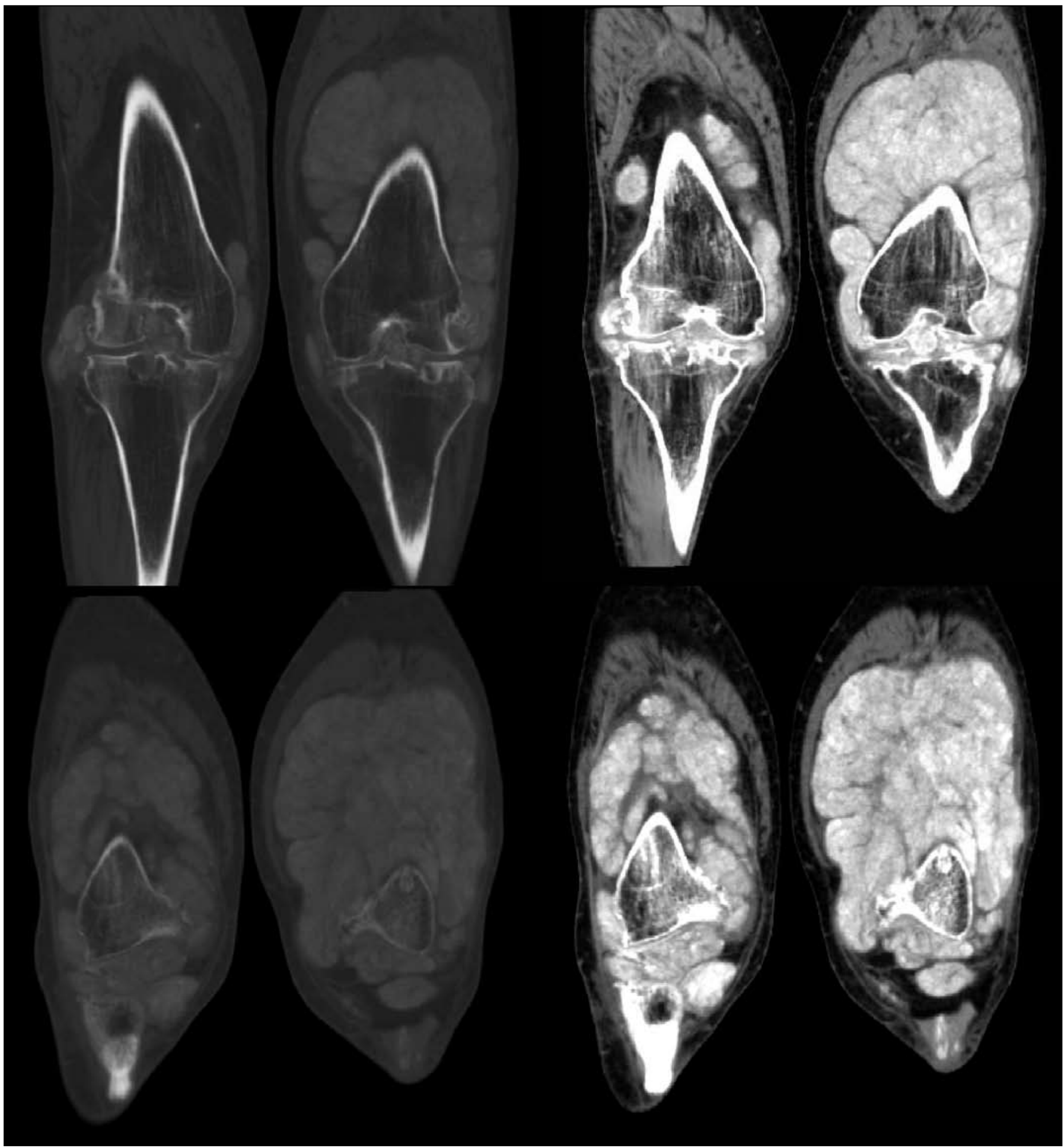

Fig. 5. Coronal non-enhanced CT images of the patient's knees show diffuse lobulated hyperdense synovial tophaceous deposits measuring 160 - 175 Hounsfield units.

\section{Imaging}

\section{Radiographs}

Radiographs remain the imaging modality of choice in the diagnosis of gouty arthritis. A characteristic feature of gout is preservation of bone mineral density until late stages of the disease; well-marginated paraarticular erosions with overhanging margins are characteristic - some of them remote from the joint. Punctate bone sclerosis may be seen owing to intra-osseous deposition of the tophi; ${ }^{2}$ with para-articular deposition of tophi, there is asymmetric soft-tissue swelling. Tophi may cause 


\section{CASE REPORT}

pressure erosion of the underlying bone. Diffuse synovial involvement around the knee, as seen in our case, has not been reported.

\section{CT}

CT has not been extensively used until recently, owing to its multiplanar capability that facilitates the recognition of para-articular calcifications when present. When not calcified, CT scans show high Hounsfield values most likely owing to high concentration of sodium nuclei in the MSU crystals. ${ }^{2.57}$ Gerster et al. have suggested that nodular lesions with Hounsfield units of 160 or above on CT may be diagnostic of gout.

\section{MRI}

The MRI appearance is variable; an inflamed joint shows effusion and para-articular oedema seen as low signal on T1- and high on T2-weighted images. During acute episodes, the para-articular structures are enhanced, following gadolinium administration. The tophaceous deposits may have a low to imtermediate signal on T1-, and a low signal (if calcified) or high signal on T2-weighted images, depending on the degree of hydrated calcium. ${ }^{25}$

\section{Conclusion}

Tophaceous gout is a rare clinical manifestation that has been observed in patients with chronic gout; diffuse involvement of the knees, as in our case, is even rarer. CT imaging, because of its speed and multiplanar capabilities, facilitates the diagnosis and assessment of tophaceous arthritis, as soft-tissue nodules of Hounsfield units above 160 may be diagnostic of gout.

1. Cornelius R, Schneider HJ. Gouty arthritis in the adult, arthritis and other arthropathies. Radiol Clin North Am 1988;26:1267-1277.

2. Monu UVM, Pope TL. Gout: A clinical and radiologic review. Radiol Clin North Am 2004;42:169-184.

3. Mandell BF. Clinical manifestations of hyperuricaemia and gout. Cleve Clin J Med 2008; 75(Suppl 5):5-8.

4. Murray RO, Jacobson HG, Stoker DJ. The Radiology of Skeletal Disorders. 3rd ed. London: Churchill Livingstone, 1990.

5. Clement KHC, Lee RY, Huay-Ben P, et al. Intra-articular gouty tophi of the knee: CT and MR imaging in 12 patients. Skeletal Radiol 1999;28:75-80.

6. Baker DL, Stroup JS, Gilstrap CA. Tophaceous gout in a patient with rheumatoid arthritis. J Am Osteopath Assoc 2007; 107:554-556.

7. Gerster JC, Landry M, Duvoisin B, Rappoport G. Computed tomography of the knee joint as an indicator of intraarticular tophi in gout. Arthritis Rheum 1996;39:1406-1409. 